\title{
ESTUDO POPULACIONAL SOBRE OS DETERMINANTES DA AUTOPERCEPÇÃO DE SAÚDE DE IDOSOS RESIDENTES EM COMUNIDADE
}

\author{
POPULATION STUDY ON THE DETERMINANTS FOR SELF-RATED \\ HEALTH OF ELDERLY RESIDENTS IN COMMUNITY
}

\author{
ESTUDIO POBLACIONAL SOBRE DETERMINANTES DE \\ AUTOPERCEPCIÓN DE SALUD EN ANCIANOS RESIDENTES EN \\ COMUNIDAD
}

\author{
Moema Santos Souza \\ Raildo da Silva Coqueiro ${ }^{* *}$ \\ Marcos Henrique Fernandes ${ }^{* * *}$
}

\begin{abstract}
RESUMO
Objetivo: Verificar os fatores determinantes da autopercepção negativa de saúde em idosos residentes em comunidade. Métodos: Trata-se de estudo transversal de base populacional do qual participaram 316 idosos residentes na zona urbana do município de Lafaiete Coutinho-Bahia. A variável dependente do estudo foi a autopercepção de saúde e as variáveis explanatórias foram distribuídas nos blocos sociodemográfico, comportamentos de risco e estado de saúde. Para análise dos dados foi realizado a regressão de Poisson com intervalo de confiança de 95\%. Resultados: A maioria dos idosos $(58,1 \%)$ percebeu sua saúde como negativa. Os resultados da análise múltipla mostraram que a autopercepção negativa da saúde foi positivamente associada a ex-fumantes, idosos com diagnóstico autorreferido de diabetes, artrite, doença pulmonar e uso de dois ou mais medicamentos. Conclusão: Os resultados do presente estudo permitiram detectar determinantes da autopercepção negativa da saúde de idosos residentes em comunidade.
\end{abstract}

Palavras chave: Autopercepção, estilo de vida, saúde do idoso, enfermagem em saúde Pública.

\begin{abstract}
Objective: To investigate the determinant factors of negative health self-concept in community-dwelling elderly. Methods: It is a population-based cross-sectional study which involved 316 elderly people living in the urban area of the municipality of Lafaiete Coutinho-Bahia, Brazil. The dependent variable was the self-rated concept of health and the explanatory variables were classified into: sociodemographic, risk behavior and health

\footnotetext{
* Enfermeira. Mestre em Enfermagem e Saúde. Professora Auxiliar, Departamento de Saúde II. Núcleo de Estudos em Epidemiologia do Envelhecimento - NEPE. Universidade Estadual do Sudoeste da Bahia - UESB, Jequié- Bahia, Brasil. E-mail: ameonsouza2@hotmail.com

** Graduado em Educação Física. Mestre em Educação Física. Professor Assistente, Departamento de Saúde I. Núcleo de Estudos em Epidemiologia do Envelhecimento - NEPE. Universidade Estadual do Sudoeste da Bahia - UESB, Jequié Bahia, Brasil. E-mail: raiconquista@yahoo.com.br

${ }^{* * *}$ Fisioterapeuta. Doutorado em Ciências da Saúde. Professor Titular, Departamento de Saúde I. Núcleo de Estudos em Epidemiologia do Envelhecimento - NEPE. Universidade Estadual do Sudoeste da Bahia - UESB, Jequié- Bahia, Brasil. Email: marcoshenriquefernandes@bol.com.br
} 
condition. For data analysis, Poisson regression was carried out, with a confidence interval of $95 \%$. Results: Most of the elderly (58.1\%) perceived their health condition as negative. The results of multivariate analysis showed that negative self-concept of health was positively associated with former smokers, elderly with selfreported diagnosis of diabetes, arthritis, lung disease, and the use of two or more drugs. Conclusion: The results of this study allowed us to detect determinants for negative health self-concept of community elderly.

Key words: Self-concept, life style, health of the elderly, nursing in public health.

\section{RESUMEN}

Objetivo: Verificar factores determinantes de autopercepción negativa de salud en ancianos residentes en comunidad. Material y métodos: Estudio transversal de base poblacional con 316 ancianos de la zona urbana del municipio de Lafaiete Coutinho - Bahia. La variable dependiente fue autopercepción de salud y las variables explicativas fueron distribuidas en: sociodemográficas, comportamientos de riesgo y estado de salud. Para el análisis de datos fue realizada la regresión de Poisson con intervalo de confianza de 95\%. Resultados: La mayoría de los ancianos $(58,1 \%)$ percibió su salud como negativa. Los resultados del análisis mostraron que la autopercepción negativa de salud fue positivamente asociada a ex fumadores, ancianos con diagnóstico autorreferido de diabetes, artritis, enfermedad pulmonar y uso de dos o más medicamentos. Conclusión: Los resultados del estudio permitieron detectar determinantes de autopercepción negativa en la salud de ancianos residentes en comunidad.

Palabras clave: Autopercepción, estilo de vida, salud del anciano, enfermería en salud pública.

Fecha recepción: 05/01/15 Fecha aceptación:12/05/16

\section{INTRODUÇÃO}

Estudos recentes entre os pesquisadores brasileiros têm apontado que o envelhecimento populacional tem ocorrido de forma mais acentuada nos países em desenvolvimento. Associado a esse crescimento, observa-se também o desafio dos múltiplos problemas de saúde, psicossociais e econômicos gerados pelo envelhecimento desordenado. Essa acentuada velocidade, colocará o pais, no período de 25 anos, como a sexta população em quantidade de idosos no mundo, em números absolutos, o que representará cerca de $15,5 \%$ da população brasileira (1).

Esse aumento no número de idosos traz também repercussões positivas e/ou negativas na forma como avaliam sua saúde. Nesse sentido, a definição de autopercepção de saú- de está baseada em uma modalidade de pesquisa qualitativa realizada através da escuta de relatos, por meio de observações, questionários ou entrevistas constituídas pela relação que o sujeito estabelece com os demais e pela forma como o sujeito é percebido pelos outros. Nas entrevistas são considerados apenas os relatos narrados pelos sujeitos, e o seu objetivo é apreender o sentido que cada pessoa atribui a sua vida (2).

Nesse sentido, a autopercepção de saúde tem sido utilizada tanto em inquéritos quanto em estudos etiológicos, tornando-se um dos indicadores mais utilizados em pesquisas na área do envelhecimento, uma vez que prediz a mortalidade e o declínio funcional, além de ser um importante indicador do estado de saúde (3).

A autopercepção de saúde e seus potenciais determinantes em idosos têm sido tema 
de pesquisa em vários países $(4,5)$. Entretanto, no Brasil, poucos estudos populacionais analisaram esse indicador em idosos residentes em comunidade, particularmente em municípios de pequeno porte com baixos indicadores de saúde (6).

Os estudos publicados $(6,7)$, vêm mostrando que os principais fatores determinantes que podem influenciar a autopercepção de saúde são os demográficos (idade, sexo, estado conjugal, e arranjo familiar), os socioeconômicos (educação e renda), o número de doenças crônicas (hipertensão, artrite ou reumatismo, doença cardiovascular, diabetes, asma, bronquite ou enfisema, embolia ou acidente vascular cerebral, e câncer), comportamentais (tabagismo, consumo de bebida alcoólica, e nível de atividade física ) e a capacidade funcional (atividades de vida diárias).

Em decorrência das diferenças geográficas, populacionais, culturais, e socioeconômicas, fazem-se necessários mais estudos relacionados à autopercepção de saúde em idosos das diferentes regiões do país. A partir da identificação dos possíveis fatores que interferem na percepção negativa de saúde por esses indivíduos será possível desenvolver ações direcionadas à saúde desse segmento populacional e atividades voltadas para prevenção de agravos e promoção da saúde com vistas a um envelhecimento ativo. Considerando a relevância da temática em questão, $o$ presente estudo teve como objetivo verificar os fatores determinantes da autopercepção negativa de saúde em idosos residentes em comunidade.

\section{MÉTODOS}

Trata-se de um estudo descritivo e de associação, com delineamento transversal, baseado em dados secundários originados da pesquisa epidemiológica, de base populacional, domiciliar, intitulada "Estado nutricional, comportamentos de risco e condições de saúde dos idosos de Lafaiete Coutinho- BA". O município de Lafaiete Coutinho, localizado no interior do nordeste do País, possui um dos piores indicadores de saúde e qualidade de vida e ocupa uma das piores colocações no ranking nacional, no Îndice de Desenvolvimento Humano (IDH) $(0,607)(8)$, no coeficiente de Ginni $(0,51)(9)$ e quanto à expectativa de vida (63,1 anos) (7). Em 2010, a população do município era de 3.901 habitantes, sendo $598(15,4 \%)$ idosos. A população do estudo foi constituída por todos os indivíduos residentes na zona urbana com idade $\geq 60$ anos $(n=355)(8)$. Destes, 316 participaram do estudo (89\%), ocorrendo $17(4,8 \%)$ recusas e $22(6,2 \%)$ perdas (indivíduos que não foram encontrados após três visitas em dias alternados).

Os dados foram coletados usando um formulário próprio, baseado no questionário SABE (Saúde, Bem Estar e Envelhecimento) (10), realizado em sete países da América Latina e Caribe, a exceção dos questionários de atividade física e de depressão que foram mais longos.

Este trabalho foi aprovado pelo Comitê de Ética em Pesquisa da Universidade Estadual do Sudoeste da Bahia (UESB), sob o número de protocolo 064/2010. A participação foi voluntária, e todos os indivíduos assinaram o Termo de Consentimento Livre e Esclarecido.

A variável autopercepção da saúde (variável dependente) foi identificada por meio do autorrelato obtida a partir do quesito "como você diria que sua saúde é?” (excelente, muito boa, boa, regular ou má), a qual posteriormente foi dicotomizada em: autopercepção da saúde positiva (excelente, muito boa e boa) e autopercepção da saúde negativa (regular e má).

As variáveis independentes foram divididas em três grupos: sociodemográficas, comportamentais e condições de saúde.

Sociodemográficas: sexo (masculino e fe- 
minino), grupo etário (60-69, 70-79 e $\geq 80$ anos), arranjo familiar (vive sozinho e acompanhado), sabe ler e escrever um recado (sim e não) e participação em atividade religiosa ( $\operatorname{sim}$ e não).

Comportamentais: tabagismo (fumante, ex-fumante e nunca fumou), consumo de bebida alcoólica (consome e não consome) e nível de atividade física, o qual foi avaliado usando o Questionário internacional de Atividade Física (IPAQ) (11), versão longa, validado e adaptado para idosos brasileiros por Manzo \& Benedetti (12) (insuficientemente ativo: menor que 150 minutos por semana em atividades físicas moderadas ou vigorosas e ativo: maior ou igual a 150 minutos por semana).

Condições de saúde: os diagnósticos de hipertensão, diabetes, câncer (exceto câncer de pele), doença crônica do pulmão, doença cardíaca, circulatória, reumatismo e osteoporose foram realizadas com questões referidas (sim; não), o uso de medicamentos (0 a 1; 2 ou mais); e hospitalização nos últimos 12 meses (nenhuma, uma ou mais vezes;). Os sintomas depressivos foram analisados pela Escala de Depressão Geriátrica GDS (13) (escore $<6$ pontos $=$ ausência de sintomas; escore $\geq 6$ pontos $=$ presença de sintomas).

Para avaliar a capacidade funcional foram utilizadas as informações do instrumento de Katz et al. (14), para atividades básicas da vida diária - ABVDs e a escala de Lawton e Brody (15) para atividades instrumentais da vida diária (AIVDs). A escala de capacidade funcional foi hierárquica, distinguindo três categorias: independente, dependente nas AIVD, dependente nas ABVD e AIVD (16).
Os idosos que relataram dependência nas ABVD, mas não nas AIVD, foram classificados na última categoria, referente à dependência em ambas as dimensões.

\section{Análise estatística}

Com o objetivo de comparar prevalências, a associação entre autopercepção de saúde e as variáveis independentes foram verificadas mediante a obtenção de estimativas brutas e ajustadas das razões de prevalências (RP), por ponto e por intervalo de confiança de 95\% (IC95\%), por meio do modelo de regressão de Poisson. Nas análises brutas, a prevalência de autopercepção negativa de saúde foi calculada para cada categoria das variáveis independentes, e o nível de significância foi testado por meio do teste de Wald para heterogeneidade. $\mathrm{Na}$ análise ajustada, foram incluídas as variáveis que apresentaram significância estatística de pelo menos $20 \%$ (p s $0,20)$ nas análises brutas, seguindo a ordem de um modelo hierárquico para determinação dos desfechos (17) como apresentado na Figura 1. De acordo com o modelo estabelecido, as variáveis dos níveis mais superiores (distais) interagem entre si e determinam as variáveis dos níveis mais inferiores (proximais). O efeito de cada variável independente sobre o desfecho foi controlado para as variáveis do mesmo nível e de níveis superiores no modelo, sendo que o critério estatístico de permanência no modelo foi de $20 \%$ ( $\mathrm{p}$ $\leq 0,20)$. As associações foram consideradas significativas, quando apresentaram nível de significância de no máximo 5\% ( $1 \leq 0,05)$. Os dados foram tabulados e analisados no IBM SPSS Statistics for Windows (IBM SPSS. 21.0, 2012, Armonk, NY: IBM Corp.). 
Nível 1: Aspectos sociodemográficos

Sexo; idade; arranjo familiar; sabe ler e escrever; atividades religiosas

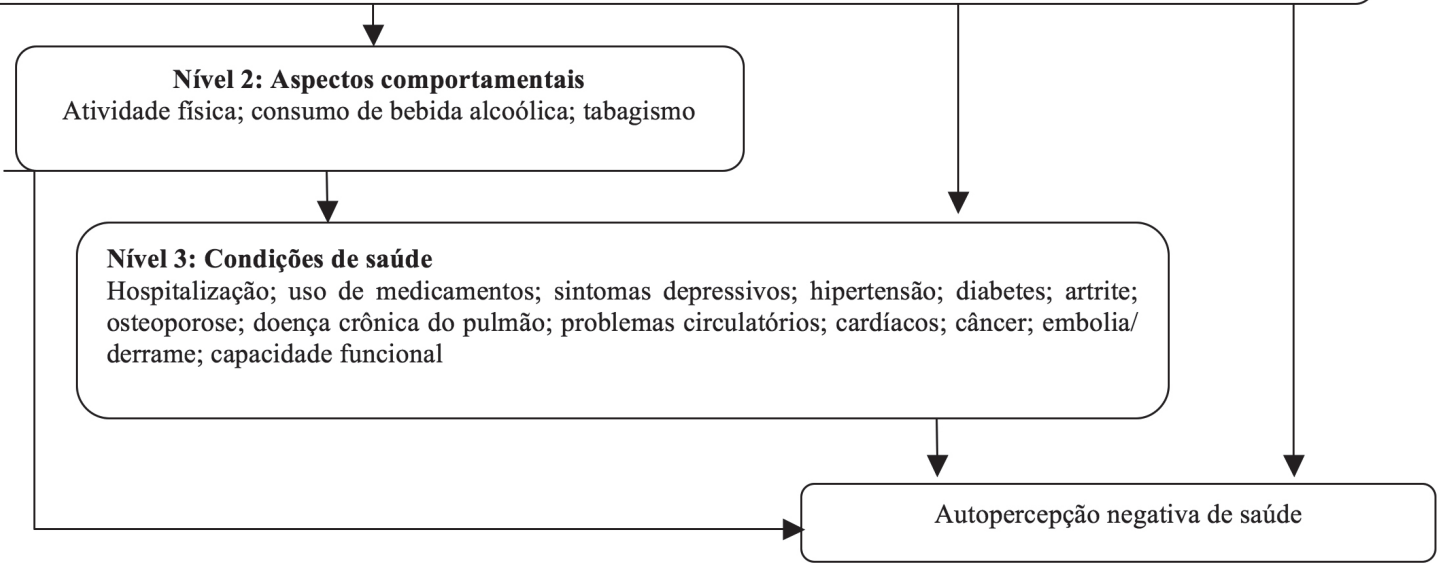

Figura 1: Modelo teórico para investigação de fatores associados a autopercepção negativa de saúde em idosos estruturado em blocos hierarquizados.

\section{RESULTADOS}

Participaram do estudo 316 idosos, sendo 173 mulheres $(54,7 \%)$ e 143 homens $(45,3 \%)$, com idade entre 60 e 105 anos e média de 74,2 $( \pm 9,8)$ anos. A idade média das mulheres foi de $74,9( \pm 10,0)$ anos e dos homens $73,4( \pm 9,4)$ anos.

A Tabela 1 mostra as características sociodemográficas e comportamentais da população estudada. Observou-se que 54,7\% dos idosos era do sexo feminino, $83,5 \%$ vivia acompanhado, 66,8\% não sabia ler ou escrever um recado e $92,4 \%$ não participava de nenhuma atividade religiosa. Com relação ao comportamento de risco, $46,7 \%$ dos idosos relataram ser ex-fumantes, $52,3 \%$ referiram ser ativos físicamente e $91,4 \%$ dos idosos não faziam uso de bebida alcoólica.

A Tabela 2 apresenta as características de condições e autopercepção de saúde dos idosos. Observou-se uma elevada proporção de idosos que referiram hipertensão $(65,6 \%)$, usavam dois ou mais medicamentos $(60,1 \%)$ e que relataram ser independentes $(42,3 \%)$.
Entretanto, também se observou um alto percentual de idosos dependente para AIVD (41,0\%). Apesar dos idosos apresentarem elevada prevalência em algumas doenças crônicas autorreferidas, foi observado um baixo percentual de hospitalização por esses indivíduos $(24,5 \%)$. A autopercepção negativa de saúde apresentou uma elevada prevalência $(58,1 \%)$.

A Tabela 3 apresenta a associação entre a autopercepção negativa de saúde e as variáveis independentes do estudo realizada pela análise de regressão bruta. Observou-se que as variáveis: sexo, tabagismo, atividade física, hospitalização, doenças crônicas autorreferidas: hipertensão, diabetes, problemas cardíacos, artrite, osteoporose, doença crônica do pulmão, sintomas depressivos, capacidade funcional e medicamentos apresentaram um nível de significância $(\mathrm{p}<0,20)$, sendo incluídas no modelo de regressão múltiplo. As demais variáveis não participaram da análise de regressão múltipla por apresentarem um nível de significância $(\mathrm{p}>0,20)$.

A Tabela 4 apresenta os resultados da análise ajustada para a autopercepção negativa 
de saúde em relação às variáveis independentes do estudo, a qual foi composta por três blocos. No primeiro bloco foram analisadas ao mesmo tempo as variáveis sociode- mográficas, de forma que nenhuma variável manteve-se associada com a autopercepção negativa de saúde.

Tabela 1. Características sociodemográficas e comportamentos de risco da população estudada. Lafaiete Coutinho, Bahia, Brasil, 2011.

\begin{tabular}{|c|c|c|c|}
\hline Variáveis & $\%$ resposta & $\mathrm{N}$ & $\%$ \\
\hline Sexo & 100 & & \\
\hline Masculino & & 143 & 45,3 \\
\hline Feminino & & 173 & 54,7 \\
\hline Grupo etário & 99,7 & & \\
\hline 60-69 anos & & 115 & 36,5 \\
\hline 70-79 anos & & 106 & 33,7 \\
\hline$>80$ anos & & 94 & 29,8 \\
\hline Arranjo Familiar & 100 & & \\
\hline Mora Sozinho & & 52 & 16,5 \\
\hline Mora Acompanhado & & 264 & 83,5 \\
\hline Sabe ler e escrever & 100 & & \\
\hline Sim & & 105 & 33,2 \\
\hline Não & & 211 & 66,8 \\
\hline Atividade religiosa & 99,4 & & \\
\hline Participa & & 24 & 7,6 \\
\hline Não participa & & 290 & 92,4 \\
\hline Atividade física & 98,1 & & \\
\hline Insuficientemente ativo & & 148 & 47,7 \\
\hline Ativo & & 162 & 52,3 \\
\hline Tabagismo & 99,7 & & \\
\hline Fumante & & 35 & 11,1 \\
\hline Ex-fumante & & 147 & 46,7 \\
\hline Nunca fumou & & 133 & 42,2 \\
\hline Consumo de bebidas alcoólicas & 99,7 & & \\
\hline Consome & & 27 & 8,6 \\
\hline Não Consome & & 288 & 91,4 \\
\hline
\end{tabular}


Tabela 2. Características do estado e autopercepção de saúde da população estudada. Lafaiete Coutinho, Bahia, Brasil, 2011.

\begin{tabular}{|c|c|c|c|}
\hline Variáveis & $\%$ resposta & $\mathrm{N}$ & $\%$ \\
\hline Hospitalização & 99,4 & & \\
\hline Uma ou mais vezes & & 77 & 24,5 \\
\hline Nenhuma & & 237 & 75,5 \\
\hline Hipertensão & 99,4 & & \\
\hline $\operatorname{Sim}$ & & 206 & 65,6 \\
\hline Não & & 108 & 34,4 \\
\hline Diabetes & 97,5 & & \\
\hline Sim & & 35 & 11,4 \\
\hline Não & & 273 & 88,6 \\
\hline Problemas Circulatórios & 99,7 & & \\
\hline $\operatorname{Sim}$ & & 20 & 6,3 \\
\hline Não & & 295 & 93,7 \\
\hline Problemas cardíacos & 99 & & \\
\hline $\operatorname{Sim}$ & & 38 & 12,1 \\
\hline Não & & 275 & 87,9 \\
\hline Artrite & 98,7 & & \\
\hline Sim & & 105 & 33,7 \\
\hline Não & & 207 & 66,3 \\
\hline Osteoporose & 95 & & \\
\hline Sim & & 22 & 7,3 \\
\hline Não & & 276 & 92,7 \\
\hline Sintomas depressivos & 91,8 & & \\
\hline Com sintomas & & 58 & 20 \\
\hline Sem sintomas & & 232 & 80 \\
\hline Câncer & 99,7 & & \\
\hline Sim & & 4 & 1,3 \\
\hline Não & & 311 & 98,7 \\
\hline Doença Crônica de Pulmão & 99,4 & & \\
\hline Sim & & 11 & 3,5 \\
\hline Não & & 303 & 96,5 \\
\hline Embolia/ Derrame & 99,7 & & \\
\hline Sim & & 20 & 6,3 \\
\hline Não & & 295 & 93,7 \\
\hline Medicamentos & 100 & & \\
\hline 0 a 1 & & 126 & 39,9 \\
\hline 2 ou mais & & 190 & 60,1 \\
\hline Capacidade funcional & 97,1 & & \\
\hline Dependente para ABVD & & 51 & 16,6 \\
\hline Dependente para AIVD & & 126 & 41 \\
\hline Independente & & 130 & 42,3 \\
\hline Autopercepção de saúde & 95,9 & & \\
\hline Positiva & & 127 & 41,1 \\
\hline Negativa & & 176 & 58,1 \\
\hline
\end{tabular}


Tabela 3. Prevalência da autopercepção negativa de saúde e análise bruta de sua associação com as variáveis independentes do estudo. Lafaiete Coutinho, Bahia, Brasil, 2011.

\begin{tabular}{|c|c|c|c|c|c|}
\hline Nivel & Variáveis & $\%$ & RPbruta & IC 95\% & $\mathrm{p}$ \\
\hline \multirow{16}{*}{1} & Sexo & & & & 0,197 \\
\hline & Feminino & 61,4 & 1,14 & $0,93-1,38$ & \\
\hline & Masculino & 54 & 1 & & \\
\hline & Idade & & & & 0,636 \\
\hline & $>80$ anos & 66,7 & 1,02 & $0,81-1,28$ & \\
\hline & 70-79 anos & 55 & 0,91 & $0,72-1,15$ & \\
\hline & $>60$ anos & 59,6 & 1 & & \\
\hline & Sabe ler e escrever um recado & & & & 0,884 \\
\hline & Não & 57,8 & 0,98 & $0,81-1,20$ & \\
\hline & Sim & 58,7 & 1 & & \\
\hline & Religião & & & & 0,433 \\
\hline & Nenhuma & 65,2 & 1,13 & $0,83-1,55$ & \\
\hline & Alguma religião & 57,5 & 1 & & \\
\hline & Arranjo Familiar & & & & 0,885 \\
\hline & Sozinho & 57,1 & 0,98 & $0,75-1,28$ & \\
\hline & Acompanhado & 58,3 & 1 & & \\
\hline \multirow[t]{10}{*}{2} & Tabagismo & & & & 0,148 \\
\hline & Fumante & 54,5 & 1,04 & $0,73-1,48$ & \\
\hline & Ex-fumante & 63,9 & 1,22 & $0,99-1,50$ & \\
\hline & Nunca fumou & 52,4 & 1 & & \\
\hline & Atividade Física & & & & 0,193 \\
\hline & Insuficientemente ativo & 61,8 & 1,14 & $0,94-1,38$ & \\
\hline & Ativo & 54,3 & 1 & & \\
\hline & Consumo de bebidas alcoólicas & & & & 0,389 \\
\hline & Consome & 48,1 & 0,81 & $0,54-1,22$ & \\
\hline & Não consome & 59,1 & 1 & & \\
\hline \multirow[t]{19}{*}{3} & Hospitalização & & & & 0,163 \\
\hline & Uma ou mais vezes & 64,9 & 1,15 & $0,94-1,41$ & \\
\hline & Nenhuma & 56,1 & 1 & & \\
\hline & Hipertensão & & & & 0,067 \\
\hline & Sim & 61,9 & 1,23 & $0,99-1,53$ & \\
\hline & Não & 50,5 & 1 & & \\
\hline & Diabetes & & & & 0,001 \\
\hline & Sim & 82,9 & 1,52 & $1,26-1,84$ & \\
\hline & Não & 54,4 & 1 & & \\
\hline & Problemas cardíacos & & & & 0,032 \\
\hline & Sim & 72,2 & 1,28 & $1,02-1,61$ & \\
\hline & Não & 56,2 & 1 & & \\
\hline & Artrite & & & & 0,001 \\
\hline & Sim & 70,3 & 1,36 & $1,13-1,63$ & \\
\hline & Não & 51,8 & 1 & & \\
\hline & Osteoporose & & & & 0,004 \\
\hline & Sim & 80 & 1,42 & $1,12-1,82$ & \\
\hline & Não & 56,1 & 1 & & \\
\hline & Sintomas depressivos & & & & 0,031 \\
\hline
\end{tabular}


Continuação Tabela 3.

\begin{tabular}{lllll}
\hline Com sintoma & 69,6 & 1,26 & $1,02-1,55$ & \\
Sem sintoma & 55,4 & 1 & & 0,735 \\
Câncer & & & & \\
Sim & 66,7 & 1,15 & $0,51-2,57$ & \\
Não & 58 & 1 & & 0,001 \\
Doença crônica do pulmão & & & & \\
Sim & 90 & 1,58 & $1,26-1,99$ & \\
Não & 56,8 & 1 & & \\
Embolia & & & & 0,149 \\
Sim & 70,6 & 1,23 & $0,89-1,70$ & \\
Não & 57,3 & 1 & & \\
Capacidade Funcional & & & & \\
Dependente ABVD & 69,8 & 1,29 & $0,99-1,65$ & \\
Dependente AIVD & 59,7 & 1,1 & $0,89-1,36$ & \\
Independente & 54,3 & 1 & & \\
Medicamentos & & & & \\
2 ou mais & 65,7 & 1,41 & $1,13-1,75$ & \\
0 a 1 & 46,7 & 1 & & \\
\hline
\end{tabular}

Tabela 4. Modelo de regressão de Poisson múltiplo hierarquizado da associação entre autopercepção negativa de saúde e as variáveis independentes do estudo. Lafaiete Coutinho, Bahia, Brasil, 2011.

\begin{tabular}{|c|c|c|c|c|}
\hline Nível & Variáveis & RPajustada & IC $95 \%$ & $\mathrm{p}$ \\
\hline \multirow[t]{3}{*}{1} & Sexo & & & 0,197 \\
\hline & Feminino & 1,14 & $0,93-1,38$ & \\
\hline & Masculino & 1 & & \\
\hline \multirow[t]{4}{*}{2} & Tabagismo & & & 0,038 \\
\hline & Fumante & 1,18 & $0,81-1,71$ & \\
\hline & Ex-fumante & 1,32 & $1,07-1,64$ & \\
\hline & Nunca fumou & 1 & & \\
\hline \multirow[t]{12}{*}{3} & Diabetes & & & $<0,001$ \\
\hline & Sim & 1,46 & $1,19-1,80$ & \\
\hline & Não & 1 & & \\
\hline & Artrite & & & 0,007 \\
\hline & $\operatorname{Sim}$ & 1,31 & $1,07-1,59$ & \\
\hline & Não & 1 & & \\
\hline & Doença do Pulmão & & & $<0,001$ \\
\hline & Sim & 1,66 & $1,31-2,11$ & \\
\hline & Não & 1 & & \\
\hline & Medicamentos & & & 0,033 \\
\hline & 2 ou mais & 1,29 & $1,02-1,64$ & \\
\hline & Até 1 & 1 & & \\
\hline
\end{tabular}


O segundo bloco foi constituído pelos comportamentos de risco, representado pelas variáveis, tabagismo e atividade física. Destacou-se como fator associado à autopercepção negativa de saúde o fato de ser ex-tabagista. A prevalência da autopercepção negativa de saúde foi $32 \%$ maior entre os ex-fumantes em relação aos que nunca fumaram. Contudo, não se observou associação significativa entre a prática insuficiente de atividade física e a autopercepção negativa de saúde.

O terceiro bloco, no qual estão agrupadas as doenças crônicas autorreferidas e uso de dois ou mais medicamentos. Os idosos que autorreferiram diabetes, artrite e doença do pulmão apresentaram maior probabilidade de autopercepção negativa de saúde $(46 ; 31$; $66 \%$, respectivamente) do que os que não referiram tais patologias. Os idosos que faziam uso de dois ou mais medicamentos apresentaram uma maior probabilidade de autopercepção negativa de saúde (29\%) do que os idosos que faziam uso de nenhum ou apenas um medicamento.

De acordo com a análise hierárquica, verificou-se que o estado de saúde (diabetes, artrite, doença do pulmão e uso de dois ou mais medicamentos) e o fato de ser ex-fumantes constituíram-se em fatores que determinaram a autopercepção negativa de saúde dos idosos.

\section{DISCUSSÃO E CONCLUSÃO}

Este estudo se propôs a identificar os fatores que são determinantes para uma autopercepção de saúde negativa em idosos residentes em uma comunidade de pequeno porte. Os principais achados desse estudo mostraram que os idosos com diabetes, artrite, doença pulmonar, os que são ex-fumantes e os que fazem uso de dois ou mais medicamentos estão associados com uma autopercepção negativa de saúde.

A prevalência de autopercepção negativa de saúde autorreferida pelos idosos foi de $58,1 \%$. Esse resultado é condizente com o estudo de Haseen et al. (18) o qual avaliou 30.427 idosos residentes na zona urbana da Tailândia e observou uma prevalência de $57 \%$.

No Brasil, resultados superiores foram encontrados em estudos desenvolvidos nas regiões Sul (60,7\%) e Nordeste (67,9\%) (4), observando uma elevada autopercepção negativa de saúde na região nordeste. Esses resultados apontam que as diferenças regionais refletem as disparidades sociais, econômicas, comportamentais e de saúde no país. Prevalências inferiores foram encontradas por outros estudos $(5,6)$, os quais também apontaram que os idosos com maior número de doenças crônicas associadas à incapacidade funcional relataram uma pior percepção de saúde.

Nesse contexto, a presente pesquisa mostra uma elevada prevalência de autopercepção negativa de saúde em idosos. Essa semelhança foi encontrada em poucos estudos desenvolvidos no Brasil $(4,6,19)$ ou em outros países (18) com a mesma faixa etária, tipo de estudo e pontos de corte. Isso demonstra a necessidade de uma maior discussão e de mais pesquisas relacionadas a essa temática, levando-se em consideração as diferenças geográficas, ambientais, e socioeconômicas em várias regiões do país para promover ações de saúde direcionadas de acordo com a especificidade de cada lugar.

No bloco de variáveis comportamentais, apenas tabagismo manteve-se associado à autopercepção negativa de saúde. Indivíduos idosos, considerados ex-fumantes, avaliaram sua saúde como negativa em maior percentual em relação aos que nunca fumaram. A relação entre idosos ex-fumantes e autopercepção negativa de saúde, observada neste estudo, também foi evidenciada em outro estudo (20). Alguns prováveis motivos são elencados para pior percepção de saúde em idosos ex-fumantes, tais como: dificuldade respiratória, declínio da capacidade funcio- 
nal e da força muscular. Apesar de não ter sido objetivo deste estudo avaliar as causas da interrupção do tabagismo, infere-se que os idosos ex-fumantes podem ter apresentado pior percepção de saúde em função de possíveis patologias que culminaram na cessação do tabagismo.

A associação entre autopercepção negativa de saúde e ex-fumantes é condizente com outro estudo (21), realizado em Belo Horizonte com dados do Projeto Bambuí, para pessoas com mais de 60 anos. Nesse estudo foi observado, especialmente nos homens, que indicadores de incapacidade funcional, doenças crônicas, como angina, infarto e artrite, e uma autoavaliação negativa da saúde estão consistentemente associados com ex-tabagistas.

A relação entre diabetes autorreferida e autopercepção negativa de saúde também foi evidenciada nesse estudo, assim como foi corroborada em outro estudo $(22,23)$ e pode ser explicada em função das limitações ou incapacidades na realização das atividades cotidianas, da dificuldade de controle da doença e das restrições alimentares, fatores que podem ter contribuído para uma pior percepção de saúde pelos idosos.

Algumas pesquisas (24) mostraram associação entre artrite autorreferida e autopercepção negativa de saúde, assim como foi observado no presente estudo. Essa relação pode ser decorrente da presença de dores articulares, muitas vezes incapacitantes, que limitam as atividades desenvolvidas pelos idosos no seu dia-a-dia, contribuindo para avaliação negativa da própria saúde, além de ser uma das mais frequentes doenças relatadas pelos idosos.

Os idosos que referiram diagnóstico de doença do pulmão apresentaram associação positiva com autopercepção negativa de saúde. De acordo com Francisco et al. (25) a prevalência de doença pulmonar revelou-se significativamente mais elevada entre os idosos que consideraram sua saúde ruim ou muito ruim. Nesse sentido, infere-se que indivíduos com problemas crônicos de saúde associados à polimorbidades tendem a avaliar sua saúde de forma negativa.

Segundo Silva et al. (26) pessoas com doença pulmonar crônica apresentam dificuldades para manter suas atividades cotidianas, o que contribui para o declínio gradual das atividades físicas, causando irritabilidade e frustração. Na visão desses autores, o indivíduo incapaz de cuidar de si mesmo tem sua autoestima e confiança abaladas, o que pode repercutir em uma percepção negativa da própria saúde.

A relação entre uso de dois ou mais medicamentos e autopercepção negativa de saúde é condizente com alguns estudos realizados no Brasil $(6,27)$. Embora o estudo de Silva et al. (6) tenha apresentado o mesmo ponto de corte, apenas o sexo feminino apresentou associação positiva com autopercepção negativa da saúde. Nesse contexto, os estudos supracitados apontam que, à medida que a população envelhece, há uma elevada prevalência de doenças crônicas. Isso, consequentemente, aumenta a quantidade de medicamentos consumidos, afetando diretamente o estado de saúde e alterando negativamente a forma como o idoso a avaliará.

De acordo com Marin et al. (28) condições frequentes entre os idosos, tais como alta prevalência de condições crônicas, uso de múltiplos medicamentos, prescrições inadequadas e problemas relacionados à automedicação, podem alterar negativamente o estado de saúde desses indivíduos.

A autopercepção é uma medida de saúde validada com dados de indicadores clínicos de morbidade e de mortalidade, além de ser um bom marcador das diferenças nos subgrupos populacionais. É importante também como ferramenta para monitorar resultados na clínica e em programas de saúde pública, visto que sua aplicabilidade no âmbito da saúde parte do pressuposto de que os sujeitos que definem o seu estado de saúde como ruim apresentam mais percepções do envelhecimento negativas do que os sujeitos que 
definiram a sua saúde como boa ou excelente (29).

Algumas considerações devem ser feitas em relação ao tipo de estudo apresentado e à existência de pesquisas voltadas para essa temática. $\mathrm{O}$ estudo transversal é restrito à identificação de associações, impossibilitando o estabelecimento da direção causal das relações encontradas, além de não permitir a realização de análises de temporalidade entre exposição e desfecho. A existência de poucos estudos envolvendo os fatores associados à autopercepção negativa de saúde em idosos residentes em municípios de pequeno porte e com baixos indicadores de saúde restringiu a comparação desses com os achados do presente estudo.

A autopercepção negativa de saúde esteve associada de maneira positiva aos idosos exfumantes, com diagnóstico autorreferido de diabetes, artrite, doença do pulmão e aos que faziam uso de dois ou mais medicamentos.

As reflexões expostas neste manuscrito sobre os fatores associados à autopercepção negativa de saúde fornecem uma visão ampla das condições de saúde dos idosos deste município e poderão subsidiar a atuação dos profissionais de saúde, tanto no ensino como na assistência, direcionada à saúde do idoso, com foco nas ações de promoção da saúde e prevenção de agravos para um envelhecimento ativo e com melhores condições de vida, além de direcionar o foco para futuros estudos.

\section{REFERÊNCIAS}

1. IBGE - Instituto Brasileiro de Geografia e Estatística: Sinopse do censo demográfico de 2010 [Internet]. Brasilia: IBGE; 2010 [citado 15 out 2011]. Disponível em: http://www.ibge.gov.br/home/estatistica/ populacao/censo2010/default_sinopse. shtm

2. Glat R, Pletsch MD. O método de história de vida em pesquisas sobre autopercepção de pessoas com necessidades educacionais especiais. Revista Educação Especial. 2009; 22(34): 139-154.

3. Blazer DG. How do you feel about...? Health outcomes in late life and selfperceptions of health and well-being. Gerontologist. 2008; 48(4): 415-22.

4. Rodrigues MA, Facchini LA, Piccini RX, Tomasi E, Thumé E, Silveira DS, et al. Uso de serviços ambulatoriais por idosos nas regiões Sul e Nordeste do Brasil. Cad Saúde Pública. 2008; 24(10): 2267-78.

5. Arnadottir SA, Gunnarsdottir ED, Stenlund $\mathrm{H}$, Lundin-Olsson L. Determinants of self-rated health in old age: A population-based, cross-sectional study using the International Classification of Functioning. BMC Public Health [Internet]. 2011 Aug [citado 23 abr 2012]; (11): 670. Disponível em: https://www.ncbi.nlm. nih.gov/pmc/articles/PMC3175467/

6. Silva RJS, Smith-Menezes A, Tribess S, Rómo-Perez V, Virtuoso Junior JS. Prevalência e fatores associados à percepção negativa da saúde em pessoas idosas no Brasil. Rev Bras Epidemiol. 2012; 15(1): 49-62.

7. Reis RH, Schwartz E, Lima LM, Lange C, Feijó AM, Duarte GC. Autopercepção de saúde de idosos usuários de um serviço de oncologia. Rev. Eletr. Enf [Internet]. 2014 jul/set [citado 20 abr 2012]; 16(3): 612-8. Disponível em: https://www.fen.ufg.br/ revista/v16/n3/pdf/v16n3a16.pdf

8. PNUD - Programa das Nações Unidas para o Desenvolvimento. Atlas de desenvolvimento humano no Brasil 2000 [Internet]. Brasil: PNUD; 2012 [citado 4 abr 2012]. Disponível em: http://www.atlasbrasil.org.br/2013/pt/perfil_m/lafaiete-coutinho_ba

9. Instituto Brasileiro de Geografia e Estatística/Censos Demográficos 1991, 2000 e 2010. Indice de Gini da renda domiciliar per capita - Bahia 2010 [Internet]. Brasilia: IBGE; 2010 [citado $10 \mathrm{fev} 2012$ ]. 
Disponível em: http://tabnet.datasus.gov. $\mathrm{br} /$ cgi/ibge/censo/cnv/giniba.def

10. Coqueiro RS, Barbosa AR, Borgatto AF. Nutritional status, health conditions and socio-demographic factors in the elderly of Havana, Cuba: data from SABE survey. J Nutr Health Aging. 2010; 14(10): 803-8.

11. Craig CL, Marshall AL, Sjöström M, Bauman AE, Booth ML, Ainsworth BE, et al. International physical activity questionnaire: 12-country reliability and validity. Med Sci Sports Exerc. 2003; 35(8): 1381-95.

12. Mazo GZ, Benedetti TRB. Adaptação do questionário internacional de atividade física para idosos. Rev Bras Cineantropom Desempenho Hum. 2010; 12(6): 480-484.

13. Almeida OP, Almeida AS. Confiabilidade da versão brasileira da escala de depressão em geriatria (GDS) versão reduzida. Arq Neuropsiquiatr. 1999; 57(2b): 421-426.

14. Katz S, Ford AB, Moskowitz RW, Jackson BA, Jaffe MW. Studies of illness in the aged. The index of ADL: a standardized measure of biological and psychosocial function. JAMA. 1963; 185(12): 914-9.

15. Lawton MP, Brody EM. Assessment of older people: self-maintaining and instrumental activities of daily living. The Gerontologist. 1969; 9(3 Part 1): 179-86.

16. Hoeymans N, Feskens EJM, Van den Bos GAM, Kromhout D. Measuring functional status: cross-sectional and longitudinal associations between performance and self-report (Zutphen Elderly Study 19901993). J Clin Epidemiol. 1996; 49(10): 1103-10.

17. Victora CG, Huttly SR, Fuchs SC, Olinto MT. The role of conceptual frameworks in epidemiological analysis: a hierarchical approach. Int J Epidemiol. 1997; 26(1): 224-227.

18. Haseen F, Adhikari R, Soonthorndhada K. Self-assessed health among Thai elderly. BMC Geriatr [Internet]. 2010 [citado 15 Mar 2011]; 10(30): 1-9. Disponível em: http://bmcgeriatr.biomedcentral.com/ articles?query $=$ Self-assessed + health + am ong + Thai+elderly\&volume $=10 \&$ search $\mathrm{T}$ ype $=\& \operatorname{tab}=$ keyword

19. Santos M, Mattos I. Condições de vida e saúde da população idosa do Município de Guaramiranga-CE. Epidemiol. Serv. Saúde, Brasília. 2011; 20(2): 193-201.

20. Freitas DHM, Campos FCA, Linhares LQ, Santos CR, Ferreira CB, Diniz BS, et al. Autopercepção de saúde e desempenho cognitivo em idosos residentes na comunidade. Rev Psiquiatr Clín. 2010; 37(1):32-5.

21. Peixoto SV, Firmo JOA, Lima-Costa MF. Condições de saúde e tabagismo entre idosos residentes em duas comunidades brasileiras (Projetos Bambuí e Belo Horizonte). Cad Saude Publica. 2006; 22(9): 1925-34.

22. Mendes TAB, Goldbaum M, Segri NJ, Barros MBA, Cesar CLG, Carandina L, et al. Diabetes mellitus: fatores associados à prevalência em idosos, medidas e práticas de controle e uso dos serviços de saúde em São Paulo, Brasil. Cad Saude Publica. 2011; 27(6): 1233-43.

23. Francisco PMSB, Belon AP, Barros MBAB, Carandina L, Alves MCGP, Goldbaum M, et al. Diabetes auto-referido em idosos: prevalência, fatores associados e práticas de controle. Cad Saude Publica. 2010; 26 (1): 175-184.

24. Pagotto V, Nakatani AYK, Silveira E. Fatores associados à autoavaliação de saúde ruim em idosos usuários do sistema Único de Saúde. Cad Saude Publica. 2011; 27(8): 1593-1602.

25. Francisco PMSB, Donalisio MR, Barros MBA, Cesar CL, Carandina L, Goldbaum M. Fatores associados a doença pulmonar em idosos. Rev Saude Publica. 2006; 40(3): 428-35.

26. Silva DMGV, Souza SS, Francioni FF, Meirelles BHS. Quality of life from the perspective of persons with chronic respiratory diseases: the contribution of a social group. Rev Lat Am Enfermagem. 
2005; 13(1): 7-14.

27. Silva AL, Ribeiro AQ, Klein $\mathrm{CH}$, Acurcio FA. Utilização de medicamentos por idosos brasileiros, de acordo com a faixa etária: um inquérito postal. Cad Saude Publica. 2012; 28(6): 1033-45.

28. Marin MJS, Cecilio LCO, Perez AE, Santella F, Silva CB, Gonçalves Filho JR, et al.
Caracterização do uso de medicamentos entre idosos de uma unidade do Programa Saúde da Família. Cad Saude Publica. 2008; 24(7): 1545-55.

29. Ron P. Elderly people's attitudes and perceptions of aging and old age: the role of cognitive dissonance? Int J Geriatr Psychiatry. 2007; 22(7): 656-62. 